\title{
Adenoviruses-mediated RNA interference targeting cytosolic phospholipase $A 2 \alpha$ attenuates focal ischemic brain damage in mice
}

\author{
HUIJUN WU, HUI LIU, FENGTONG ZUO and LIHUA ZHANG \\ Department of Neurology, Cangzhou Central Hospital, Cangzhou, Hebei 061001, P.R. China
}

Received October 28, 2016; Accepted June 19, 2017

DOI: $10.3892 / \mathrm{mmr} .2018 .8610$

\begin{abstract}
Cerebral ischemia injury is a clinical, frequently occurring disease, which causes a heavy burden on society and families. It has been demonstrated that cytosolic phospholipase $\mathrm{A} 2 \alpha(\mathrm{cPLA} 2 \alpha)$ is significant in neurological injury caused by ischemic brain injury, and inhibition of cPLA $2 \alpha$ may reduce stroke injury. In the present study, the role of cPLA $2 \alpha$ was investigated in a mouse model of middle cerebral artery occlusion and/or reperfusion (MCAO/R) using an effective cPLA2 $\alpha$ inhibitor and adenoviruses-mediated RNA interference. The mosteffective recombinant adenovirus encoding cPLA $2 \alpha$ small interfering RNA (pAd-siRNA-cPLA2 $\alpha$ ) was constructed and selected. MCAO/R surgery is used to construct the model of focal ischemic brain damage in mice. Adenoviruses-mediated RNA interference targeting cPLA $2 \alpha$ was administered by stereotactic surgery $2 \mathrm{~h}$ before the MCAO/R. The expression/activity of cPLA $2 \alpha$ and cPLA $2 \alpha$-derived injurious lipid mediators was assessed. pAd-siRNA-cPLA2 $\alpha$-treated animals (RNA interference; RNAi group) were compared with pAd-siRNA-control-treated animals (negative group) with regard to neurological deficit, motor function, pathological changes, apoptosis, and infarct volume. The RNAi group animals reduced the expression level of cPLA $2 \alpha$, as determined by western blotting and reverse transcription-quantitative polymerase chain reaction, the improvement of locomotor function was evaluated by rotarod test, and the decrease of apoptosis was evaluated by terminal deoxynucleotidyl transferase dUTP nick end-labeling staining. The decreased infarct areas were evaluated by 2,3,5-triphenyltetrazolium chloride staining. The expression levels of prostaglandin E2, leukotrienes $\mathrm{B} 4$, lysophosphatidylcholine and free fatty acids were reduced in the RNAi group when compared with the negative
\end{abstract}

Correspondence to: Mrs. Lihua Zhang, Department of Neurology, Cangzhou Central Hospital, 16 Western Xinhua Road, Cangzhou, Hebei 061001, P.R. China

E-mail: zlhzxyy@126.com

Key words: ischemic brain damage, RNA interference, cytosolic phospholipase A2 $\alpha$, middle cerebral artery occlusion and/or reperfusion, terminal deoxynucleotidyl transferase dUTP nick end-labeling staining, neurological deficit score control group. Thus, the data indicates that the expression level of cPLA $2 \alpha$ was effectively controlled by pAd-siRNA-cPLA2 $\alpha$ treatment. pAd-siRNA-cPLA $2 \alpha$ treatment, in reducing the levels of inflammatory factors, neurological deficit and tissue damage, represents an effective potential therapeutic strategy. pAd-siRNA-cPLA $2 \alpha$ reduces cPLA $2 \alpha$ expression levels with long-term efficacy, thereby improving functional deficits and effectively attenuating ischemic brain damage. Thus, pAd-siRNA-cPLA $2 \alpha$ shows potential value for therapeutic evaluation in ischemic brain damage.

\section{Introduction}

Ischemic brain damage has long been associated with neuronal injury (1-4), and it is a result of combined action of acute necrosis with delayed injury mechanisms. The damage cascade is the recognized theory of the cerebral ischemia physiological and pathological mechanism, predominantly including excitotoxicity, peri-infarct depolarization, inflammation and apoptosis (5). Metabolism of arachidonic acid (AA) and its metabolic products have been implicated to be associated with these pathophysiological mechanisms (4). To a great extent, cellular levels of AA are under the control of phospholipase A2 $\left(\mathrm{PLA}_{2}\right)$ enzymes. PLA 2 catalyze the membrane phospholipid hydrolysis, and the resulting released AA is metabolized into eicosanoids. PLA 2 is a family of esterases that catalyze the hydrolysis of phospholipids in the sn-2 position, and it is the crucial enzyme of AA, prostaglandin (PG) and platelet activating factor (PAF) (6). Previous studies have indicated that $\mathrm{PLA}_{2}$ is crucial in ischemic cell death $(1,7,8)$.

Almost all human cells contain $\mathrm{PLA}_{2}$, and numerous isotypes have been identified. There are three well known major subfamilies: Secretory PLA $2, \mathrm{Ca}^{2+}$-independent $\mathrm{PLA}_{2}$ and cytosolic $\mathrm{Ca}^{2+}$-dependent PLA2 (cPLA $)$. The $\operatorname{cPLA}_{2} \alpha(\mathrm{cPLA} 2 \alpha)$ form is a member of the $\mathrm{CPLA}_{2}$ class, and has unique characteristics, including its preference to catalyze the hydrolysis of sn-2 position of phospholipid substrate molecules to AA. Numerous studies demonstrated that cPLA2 $\alpha$ is a key component associated with the development of stroke injury $(2,3,9,10)$, and it has been shown that cPLA $2 \alpha$ has a marked role in neurological injury caused by ischemic brain injury, and the inhibitor of cPLA2 $\alpha$ may reduce stroke injury (11).

RNA interference (RNAi) is an experimental technique with a wide range of applications in gene function research and gene therapy, and there is increasing focus on developing 
therapeutic strategies based on RNAi (12). Since the discovery of mammalian RNAi, $>50,000$ studies have described the use of small interfering RNA (siRNA) (13). RNAi is a gene silencing mechanism triggered by double-stranded RNA with 9-23 base pairs, it activates an RNA-induced silencing complex (RISC) and guides the degradation of homologous RNA by functional RISC (13). Compared with other antisense strategic tools, RNAi is a perfect technique for gene silencing with sensitivity and specificity (14).

Silencing of target genes in dividing and non-dividing cells is effectively via RNAi technology (15). In mammalian cells, RNAi efficiently blocks specific gene expression to treat diseases caused by excess protein expression. Due to the poor efficiency of retrovirus vectors and liposomes in transfection, adenovirus-mediated gene transfer was developed (16). The transfection capacity of adenovirus vectors was identified to be highly effective in vivo and in vitro. The expression of foreign genes in brain tissues peaked 7 days after transfection and terminated after 2 weeks (17). It has been demonstrated that a low degree virus, limited within a certain range, prevents the body's immune response. Low dosage adenovirus transfection to ischemic brain tissue with is efficient and stable with low toxicity. To the best of our knowledge, there are no studies reporting the effects of cPLA $2 \alpha$ RNAi on the development of ischemic brain damage in an in vivo animal model. Therefore, the present study employed the adenovirus-delivered RNAi technique to evaluate the effects of cPLA $2 \alpha$ knockdown in vivo in a mouse model, and hypothesized that it may be used in further adenovirus intervention experimental research.

\section{Materials and methods}

Cell culture. L929 and HEK293 cells were purchased from Shanghai Bioleaf Biotech Co., Ltd. (Shanghai, China). Cells were maintained in Dulbecco's modified Eagle's medium (DMEM) containing 10\% fetal bovine serum (both from Gibco; Thermo Fisher Scientific, Inc., Waltham, MA, USA) and $100 \mathrm{U} / \mathrm{ml}$ penicillin/streptomycin (AppliChem $\mathrm{GmbH}$, Darmstadt, Germany). At confluency, cells were split $~ 1: 3$. Cultures were incubated at $37^{\circ} \mathrm{C}$ in a humidified atmosphere with $5 \% \mathrm{CO}_{2}$. The growth media was replaced every other day. The engineered, stable L929-cPLA2 $\alpha$-siRNA cells were maintained in the above-mentioned conditions. The L929 cells stably expressed wild-type human cPLA $2 \alpha$.

Reagents and siRNA. If not otherwise stated, reagents were supplied by Sigma-Aldrich (Merck KGaA, Darmstadt, Germany). Arachidonyl trifluoromethyl ketone (ATK), an effective inhibitor of $\mathrm{cPLA}_{2}$, was purchased from Cayman Chemical Co. (Ann Arbor, MI, USA). The siRNAs targeting the mouse cPLA2 $\alpha$ gene and the sequences of RNAi were designed and synthesized by Guangzhou RiboBio Co., Ltd. (Guangzhou, China). Three gene sequences were selected for RNAi, which were verified by experiments to suppress the expression of mouse cPLA2 $\alpha$ in L929 cells (18). The siRNA sequences (sense strand) were as follows: No. 1, 5'-GCGAAC GAGACCTTCAAT-3'; no. 2, 5'-GGTGCATAACTTCAT GCTG-3'; no. 3, 5'-GCACATCGTGAGTAATGAC-3'. 5'-TTC TCCGAACGTGTCACGT-3' served as a negative control. A BLAST search on the NCBI website was used to confirm that the sequences did not have homology to any other mouse genes.

Generation and appraisal of recombinant adenovirus encoding cPLA2 $\alpha$ siRNA ( $p A d-$ siRNA-cPLA2 $\alpha$ ). To construct a plasmid expressing siRNA/cPLA $2 \alpha$, the target sequences were subcloned into shuttle vector, pShuttle-H1, then sequencing was performed by Thermo Fisher Scientific, Inc. (19). The replication-deficient adenovirus that contained the full-length cDNA of the RNAi sequence was generated by homologous recombination through co-transfection of plasmid, pShuttle-H1-RNAi with pAdEasy into HEK293 cells that were grown in DMEM to $70 \%$ confluence in $60 \mathrm{~mm}$ wells using DOTAP liposome reagent. The cells were incubated for $48 \mathrm{~h}$ at $37^{\circ} \mathrm{C}$. Following several rounds of plaque assay for purification (20), the adenovirus was purified from cell lysates via banding twice in $\mathrm{CsCl}$ density gradients. The harvested viral supernatant was desalted and added to phosphate-buffered saline (PBS) containing 10\% glycerol (v/v). The infectious titer was determined via a standard plaque assay and the titers averaged $2 \times 10^{9} \mathrm{pfu} / \mathrm{ml}$. The mRNA and protein expression levels were detected by reverse transcription-quantitative polymerase chain reaction and western blot analyses.

RNAi target screening. At $24 \mathrm{~h}$ prior to transfection, L929 cells (90\% confluent) were digested for $5 \mathrm{~min}$ at $37^{\circ} \mathrm{C}$ with trypsin/EDTA solution (Invitrogen; Thermo Fisher Scientific, Inc.). The cells were inoculated in 12-well tissue culture plates with $1.5 \mathrm{ml}$ cell culture medium (DMEM containing 10\% FBS and $100 \mathrm{U} / \mathrm{ml}$ penicillin/streptomycin) and $5 \times 10^{5}$ cells/ well, and incubated in culture medium overnight at $37^{\circ} \mathrm{C}$. The cells were transfected with Ad-siRNA-cPLA $2 \alpha$ or negative control (NC) for detecting the efficiency of silencing. The transfection efficiency of the adenovirus had plateaued at 7 days post-transfection, and cPLA $2 \alpha$ protein and mRNA expression levels were analyzed by western blotting and RT-qPCR 4 days after transfection. All transfections were performed in triplicate.

Animals and treatment. Experimental mice (male C57BL/6 mice, aged 12-16 weeks) were purchased from Better Biotechnology Co., Ltd. (Jiangsu, China). The mice were housed in a constant-temperature facility with a 12-h dark/light cycle and given access to water and food freely. All procedures performed with mice were according to the National Institutes of Health Guide for the Care and Use of Laboratory Animals (21), and the experimental procedures were approved by the Ethics Committee of Hebei, Cangzhou Central Hospital (Cangzhou, China).

The mice were randomly allocated to the sham, middle cerebral artery occlusion and/or reperfusion (MCAO/R), NC and RNAi groups. At $2 \mathrm{~h}$ before unilateral ischemia, freshly prepared pAd-siRNA-cPLA $2 \alpha$ and pAd-siRNA-control were injected into the RNAi and NC animals, respectively. The sham and $\mathrm{MCAO} / \mathrm{R}$ group animals received only the carrier solution. Mice were sacrificed by cardiac puncture following anesthetization by intraperitoneal injection of pentobarbital sodium $(45 \mathrm{mg} / \mathrm{kg}) 2 \mathrm{~h}, 1,3,7$ or 14 days post-surgery. Blood $(500 \mu \mathrm{l})$ was collected by direct cardiac puncture and indomethacin $(10 \mu \mathrm{M})$ was added to inhibit PG synthase activity 
immediately after the blood was drawn. Brains were removed from the skull and prepared for the experience. Brain tissue samples from each group were divided into two parts, some ( $n=3$ of each group) were fixed in $4 \%$ paraformaldehyde in PBS, and the others ( $n=3$ of each group) were stored at $-80^{\circ} \mathrm{C}$ until use. At 14 days post-surgery, the mice $(n=3$ from each group) were anesthetized and perfused through the left ventricle with 0.01 M PBS. Brains were removed from the skull and the rhinencephalon, cerebellum and brain stem were removed. The coronary brain regions of brain tissue samples were divided into slices ( $2 \mathrm{~mm}$ thick) and prepared for triphenyltetrazolium chloride (TTC) staining.

Stereotactic surgery. Prior to the MCAO/R surgery, animals were anaesthetized with $2 \%$ halothane in a mixture of $60 \%$ $\mathrm{N}_{2} \mathrm{O}$ and $40 \% \mathrm{O}_{2}$ using a face mask and fixed in stereotactic apparatus, where a longitudinal incision was made in the middle of the head. The skull was drilled into and the epidural thorn was opened. A microsyringe (World Precision Instruments, LLC, Sarasota, FL, USA) was used to slowly puncture to a corresponding depth (0.4-0.6 mm), and pAd-siRNA-cPLA2 $\alpha$ and pAd-siRNA-control, were injected at a constant slow speed $(200 \mathrm{nl} / \mathrm{min}$; dose, $200 \mathrm{nl})$. The wounds were stitched following surgery.

Focal cerebral ischemia model. The transient focal ischemic brain damage in mice was performed using the intraluminal occlusion technique, as described previously (22). Occlusion of the right MCA was removed after $1 \mathrm{~h}$, and the surgery in the sham group mice was performed through a vertical cervical incision. Thermostatically controlled heating pads and a heating lamp were used to maintain a normal body temperature until the mouse recovered from anesthesia. In addition, a laser Doppler perfusion monitor was used to monitor the regional blood flow. The cerebral blood flow $(\mathrm{CBF})$ measurements in the mice were performed with the probe affixed with glue to the surface of the cerebral cortex perfused by the MCA.

Neurological deficit in the mice was evaluated using a 5-point neurological deficit score (NDS) following surgery and prior to euthanasia. Neurological impairment was assessed using the scores between 0 and 4 as follows: 0 , no neurological deficit; 1 , forelimb weakness; 2 , circling to the affected side; 3 , falling to the affected side; and 4 , unable to walk spontaneously (23). Animals that were scored 1-3 following reperfusion were used for subsequent experiments, and the required number of mice were randomly selected to the experimental group. The mice were scored on day 1, 3, 7 and 14 day after reperfusion.

Evaluation of locomotor function. The rotarod test was used to analyze the motor coordination and anti-fatigue characteristics of the mice. The rotarod machine (Med Associates, Inc., Fairfax, VT, USA) adjusts the initial rotating and accelerating speeds. The detecting parameters in the present study were the latency time to the first fall and the number of falls in $5 \mathrm{~min}$. The experimental mice were trained at $2-20 \mathrm{rpm}$ in the days prior to commencing testing. Assessment of their motor ability was performed 1 day before surgery and on days 1, 3, 7 and 14 after surgery.
Enzyme-linked immunosorbent assay (ELISA). The levels of leukotriene B4 (LTB4) and prostaglandin E2 (PGE2) were examined using commercially-available ELISA kits (cat nos. 10009292-96S and 500141-96; Cayman Chemical Co.). The experiment required $50 \mu \mathrm{l}$ serum samples from the mice, and all procedures were performed according to the manufacturer's instructions.

High-performance thin-layer chromatography (HPTLC). Free fatty acids (FFAs) and lysophosphatidylcholine (LPC) were determined and quantified using HPTLC. The required lipids samples were extracted from the brain tissue samples using the Folch method (24). FFA and LPC levels were determined and quantified using HPTLC plates according to previously described methods $(25,26)$.

Histological examination. The tissue samples were fixed and embedded in paraffin ( $4 \mu \mathrm{m}$ sections) and processed in an alcohol series (50-100\%), stained with hematoxylin for $10 \mathrm{~min}$ and eosin for $5 \mathrm{~min}$ at $25^{\circ} \mathrm{C}(\mathrm{H} \& \mathrm{E})$. The stained sections were observed under inverted microscope (magnification, x100) for histological examination.

Terminaldeoxynucleotidyl transferase dUTP nick end-labeling (TUNEL) staining. To evaluate the degree of apoptosis, TUNEL staining was performed on brain sections using a TUNEL Apoptosis Assay kit (cat. no. 11684795910; Roche Diagnostics, Basel, Switzerland). The tissue samples were fixed in $4 \%$ paraformaldehyde for $24 \mathrm{~h}$ at $4^{\circ} \mathrm{C}$, paraffin-embedded, dewaxed and the TUNEL assay was performed. All procedures were conducted according to the manufacturer's instructions. DAPI Fluoromount- $\mathrm{G}^{\mathrm{TM}}$ anti-fluorescence quenching sealant (cat. no. 36308ES11; Shanghai Yeasen Biotechnology Co., Ltd., Shanghai, China) was used to seal slices. The observed results were recorded using a fluorescence microscope equipped with AxioVision software (version 4.1; Carl Zeiss AG, Oberkochen, Germany). Four brain samples from each experimental group were used for the analysis. At least four microscopic fields within the ischemic penumbra of each brain sample were imaged, and a double-blinded manner was used to count the TUNEL-positive cells. The mean value of the TUNEL-positive cells counted from these areas was calculated to represent each brain section.

Western blotting. Total proteins were extracted from the cells or frozen tissue samples using radioimmunoprecipitation assay lysis buffer (Sigma-Aldrich; Merck KGaA). The brain tissue lysates were prepared as described previously (27). Briefly, brain tissue samples were homogenized, centrifuged and the supernatants were collected. The Bradford method was used to determine the protein concentrations (28), and bovine serum albumin standard protein served as the standard. Western blotting was performed according to standard protocols. The proteins $(40 \mu \mathrm{g})$ were separated by $12 \%$ SDS-PAGE, then transferred to a polyvinylidene fluoride membrane and blocked with $5 \%$ non-fat dried milk at $37^{\circ} \mathrm{C}$ for $2 \mathrm{~h}$. Membranes were incubated with the following primary antibodies for $16 \mathrm{~h}$ at $4^{\circ} \mathrm{C}$ : Anti-cPLA2 $\alpha$ antibody (cat. no. sc-137069; 1:200; Santa Cruz Biotechnology, Inc., Dallas, TX, USA) and anti- $\beta$-actin antibody (cat. no. sc-8432; 1:200; Santa Cruz Biotechnology, 
Inc.). The membrane was subsequently incubated with rabbit anti-mouse IgG secondary antibody (cat. no. A9044; 1:500; Sigma-Aldrich; Merck KGaA) at $37^{\circ} \mathrm{C}$ for $1 \mathrm{~h}$. Bands were visualized with an enhanced chemiluminescence visualization reagent (cat. no. GERPN2232; Sigma-Aldrich; Merck KGaA). The protein bands were quantified using Quantity One software (version 4.4.0.36; Bio-Rad Laboratories, Inc., Hercules, CA, USA).

$R N A$ isolation and $R T-q P C R$. Total RNA extraction, and RT-qPCR were performed as previously described (29). Total RNA was isolated from the tissue samples or cells using TRIzol reagent (Invitrogen; Thermo Fisher Scientific, Inc.) according to the manufacturer's instructions. First-strand cDNA was synthesized using PrimeScript reverse transcriptase (Takara Biotechnology Co., Ltd., Dalian, China) and oligo (dT). Following RT, qPCR was performed according to the manufacturer's instructions of the SYBR Premix Ex Taq ${ }^{\mathrm{TM}}$ II PCR kit (Takara Bio, Inc., Otsu, Japan). The thermocycling conditions were as follows: $94^{\circ} \mathrm{C}$ for $3 \mathrm{~min}$, followed by 40 amplification cycles of $30 \mathrm{sec}$ at $94^{\circ} \mathrm{C}, 30 \mathrm{sec}$ at $58^{\circ} \mathrm{C}$ and $30 \mathrm{sec}$ at $72^{\circ} \mathrm{C}$. The relative expression levels of cPLA2 $\alpha$ mRNA were calculated using SYBR green on the ABI Prism 7300 Sequence Detection System (Applied Biosystems; Thermo Fisher Scientific, Inc.) using $\beta$-actin as the endogenous reference gene amplified from the samples. The relative amounts of mRNA were calculated using the $2^{-\Delta \Delta \mathrm{Cq}}$ method (30). The primers were as follows: cPLA2 $\alpha$ forward, 5'-GGTGGGAGAGAAGAAAGAAGTC-3' and reverse, 5'-AGGGATTTTGGATTGTGCGACC-3'; $\beta$-actin forward, 5'-GCTATGCTCTCCCTCACGCCAT-3' and reverse, 5'-TCACGCACGATTTCCCTCTCAG-3'.

TTC staining. The infarct volume was evaluated by TTC staining as previously described (31). The $2 \mathrm{~mm}$ brain sections were incubated with $2 \% \mathrm{TTC}$ at $37^{\circ} \mathrm{C}$ for $30 \mathrm{~min}$ with gentle shaking, then were fixed with $10 \%$ formalin in PBS. The stained slices were photographed, and the size of the infarct volume was determined by subtracting the area of the non-infarcted ipsilateral hemisphere from that of the intact contralateral hemisphere. The percentage of infarct volume was calculated by dividing the sum of the area from all sections of infarction by the total of that of contralateral hemisphere to avoid the influence of tissue edema (32).

Statistical analysis. All values are presented as the mean \pm standard deviation. Each experiment was repeated at least three times. Statistical analysis was performed by comparing two groups using Student's t-test and $\mathrm{P}<0.05$ was considered to indicate a statistically significant difference.

\section{Results}

Selection of RNAi sequence for knockdown of mouse cPLA2 $\alpha$ in L929 cells. We examined the inhibitory effects of the three RNAi sequences for mouse cPLA2 $\alpha$ in L929, and attempted to establish the most effective silencer. ATK effectively inhibited cPLA $2 \alpha$ in vitro and in vivo (33-37). The treatment of L929 cells with $10 \mathrm{nM}$ ATK every $24 \mathrm{~h}$ served as a positive control. The level of cPLA $2 \alpha$ in L929-cPLA $2 \alpha-$ RNAi cells was examined by qPCR and western blotting. The effects

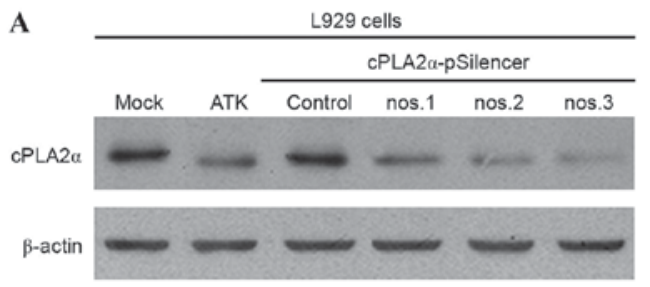

B

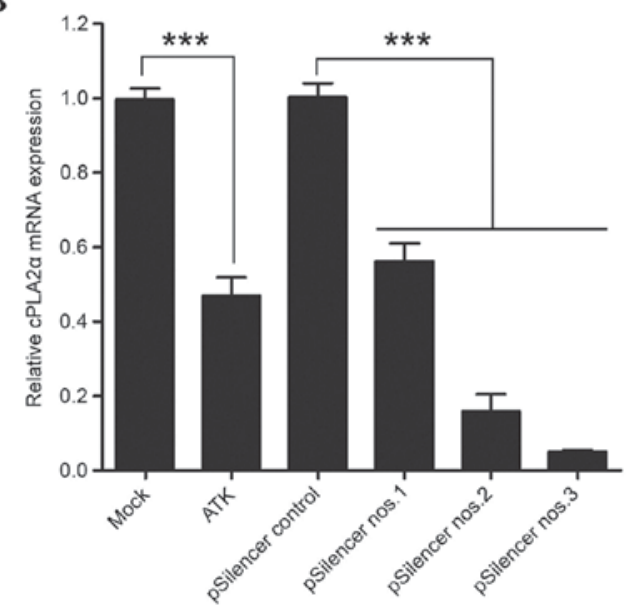

Figure 1. Effects of siRNAs for mouse cPLA $2 \alpha$ on expression levels of cPLA2 $\alpha$ in L929 cells. L929 cells were transfected with Ad-siRNA-cPLA2 $\alpha$ (pSilencer nos. 1-3) or negative control, L929 cells were treated with $10 \mathrm{nM}$ ATK every $24 \mathrm{~h}$ as a positive control. Mock group cells were treated with the same quantity of ATK solvent solution at the same time. (A) The protein expression levels of cPLA $2 \alpha$ in the different groups, with $\beta$-actin serving as the control. (B) mRNA expression levels of cPLA2 $\alpha$. ${ }^{* * *} \mathrm{P}<0.001$ (Student's t-test). siRNA, small interfering; cPLA $2 \alpha$, cytosolic phospholipase A2 $\alpha$; ATK, arachidonyl trifluoromethyl ketone.

of three predesigned RNAi sequences for mouse cPLA2 $\alpha$ (silencer nos. 1-3) on cPLA2 $\alpha$ protein and activity (Fig. 1) were confirmed. Treatment with silencer- 2 or -3 decreased the expression level and activity of cPLA $2 \alpha$ markedly, while Silencer-1 only exerted a partial inhibitory effect. Results showed that cells under silencer- 3 treatment almost entirely inhibited the expression level of cPLA $2 \alpha$ protein without altering $\beta$-actin expression. These results indicate that the Silencer-3 sequence is an effective RNAi sequence for inhibiting mouse cPLA2 $\alpha$ expression. Subsequently, the most effective RNAi sequence was used to knockdown the expression of cPLA $2 \alpha$ in mice.

Neurological function and motor function evaluation in animals following $M C A O / R$ surgery. To investigate the role of cPLA $2 \alpha$ in focal ischemic brain damage, $\mathrm{MCAO} / \mathrm{R}$ surgery was performed on male C57BL/6 mice (age, 12-16 weeks). Neurological deficits of these mice were assessed and $2 \mathrm{~h}$ after reperfusion, all mice exhibited neurological injury. Neurological deficit was evaluated again on days 1, 3, 7 and 14 after reperfusion. The RNAi group demonstrated no significant difference compared with the NC group and $\mathrm{MCAO} / \mathrm{R}$ group at $2 \mathrm{~h}$ post-surgery $(\mathrm{P}>0.05)$; however, the NDSs of the RNAi group animals were significantly lower than those of the control group mice at 7 and 14 days after stroke (Fig. 2B; $\mathrm{P}<0.01$ ).

Ischemic brain damage patients are prone to motor function impairment, losing partial or complete motor ability 

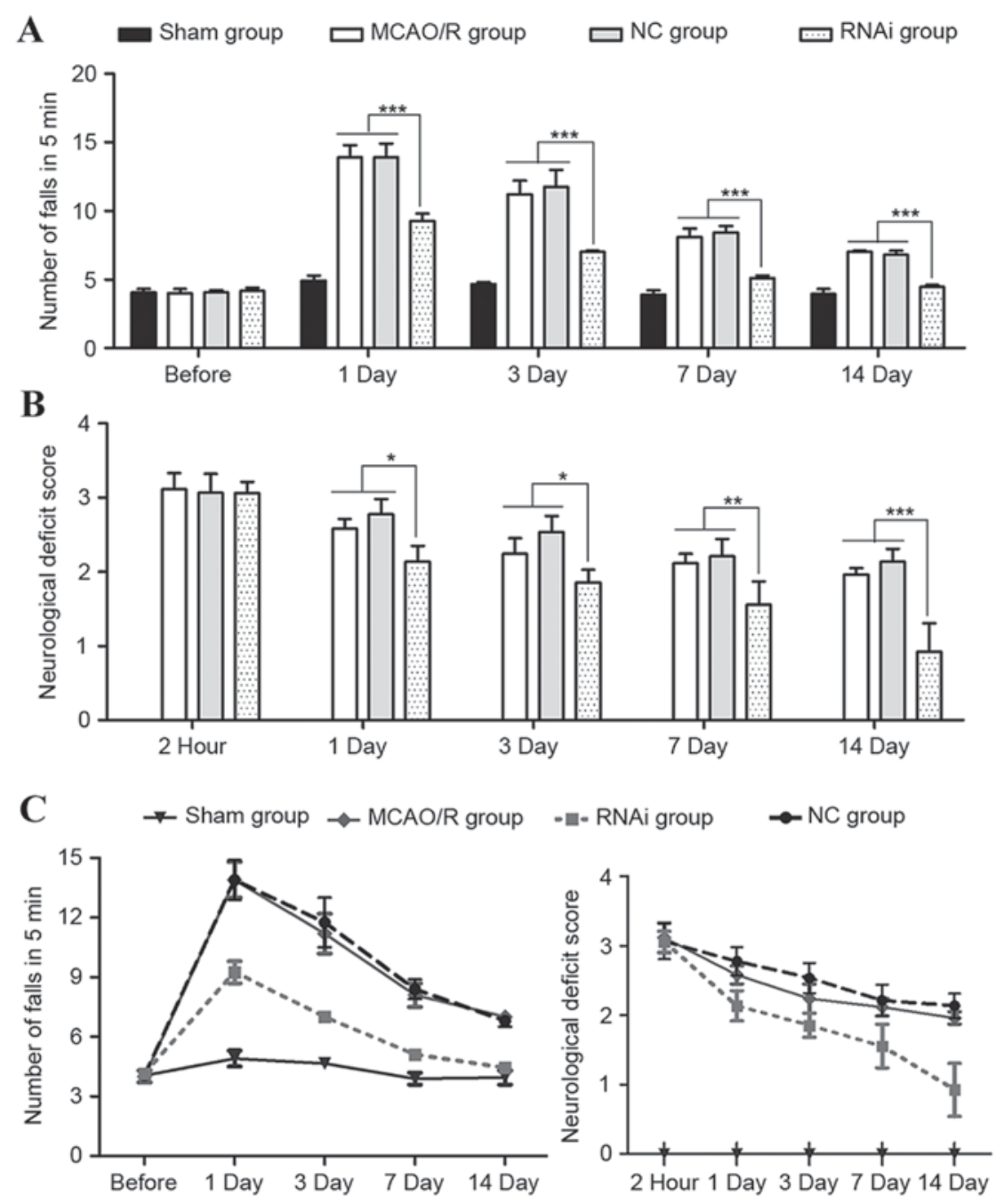

Figure 2. Motor dysfunction and neurological deficit in mice before and after MCAO/R. (A) The mean number of mouse falls in the same group in 5 min measured during the rotarod test. (B) Assigned neurological deficit scores at $2 \mathrm{~h}$, day 1, 3, 7 and 14 after MCAO/R surgery. (C) Tendency of neurological function dynamic changes in the different groups. Data are presented as the mean \pm standard error of the mean. ${ }^{*} \mathrm{P}<0.05,{ }^{* *} \mathrm{P}<0.01$ and ${ }^{* * * *} \mathrm{P}<0.001\left(\mathrm{Student}{ }^{\prime}\right.$ t-test). MCAO/R, middle cerebral artery occlusion and/or reperfusion; NC, negative control; RNAi, RNA interference.

(clinically termed paralysis). To monitor the motor function change of mice following ischemic stroke, rotarod tests were performed at 1, 3, 7 and 14 days after ischemic stroke surgery. Prior to surgery, the mice demonstrated no differences during the rotarod test (Fig. 2). However, the number of falls in 5 min was significantly higher in the MCAO/R surgery mice compared with the sham mice when evaluated following surgery (Fig. 2A). Seven days after surgery, the RNAi group exhibited a good recovery as assessed by the number of falls in $5 \mathrm{~min}$, whereas the $\mathrm{MCAO} / \mathrm{R}$ group and $\mathrm{NC}$ group mice continued to demonstrate evident deficits at that time-point. These data indicate that pAd-siRNA-cPLA $2 \alpha$ treatment in mice alleviates exacerbated motor dysfunction induced by ischemic brain damage.

Effects of pAd-siRNA-cPLA2 $\alpha$ on the expression levels of cPLA2 $\alpha, F F A, L P C, P G E 2$ and LTB4. The role of cPLA $2 \alpha$ in exacerbating transient focal ischemic brain damage has been previously demonstrated in cPLA $2 \alpha$-knockout mice (11). In the present study, whether the use of pAd-siRNA-cPLA2 $\alpha$ attenuates ischemic brain damage was evaluated in a mouse model of $\mathrm{MCAO} / \mathrm{R}$. A laser Doppler perfusion monitor was used to monitor the regional blood flow and this was continued until $20 \mathrm{~min}$ after reperfusion. No difference in blood flow was detected in the mice from the different groups at any time (Fig. 6). Compared with the NC group, the RNAi group effectively decreased the cPLA $2 \alpha$ expression levels in the ischemic damage mice at $2 \mathrm{~h}, 1,3$ and 7 days after surgery (Fig. 3; P<0.01).

To evaluate whether pAd-siRNA-cPLA $2 \alpha$ treatment decreased the levels of phospholipid degradation products, cPLA $2 \alpha$-derived injurious lipid mediators in the brain tissue samples were assessed. The results indicated that pAd-siRNA-cPLA2 $\alpha$ treatment significantly reduced the levels of FFA and LPC, while the levels of FFA and LPC exhibited a marked increase in the $\mathrm{NC}$ group (Fig. 4A and B). At the measurement time-points, the levels of FFA and LPC in the RNAi group did not recover to the normal levels of the sham group (data not shown). $\mathrm{cPLA}_{2}$ tend to catalyze the hydrolysis of phospholipids in the sn-2 position (35), and it is the crucial enzyme of AA, PG and PAF. PGE2 and LTB4 are proinflammatory eicosanoids metabolized from free AA. Serum from 
A

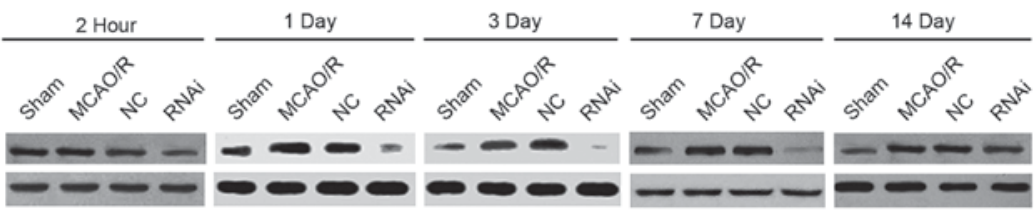

B
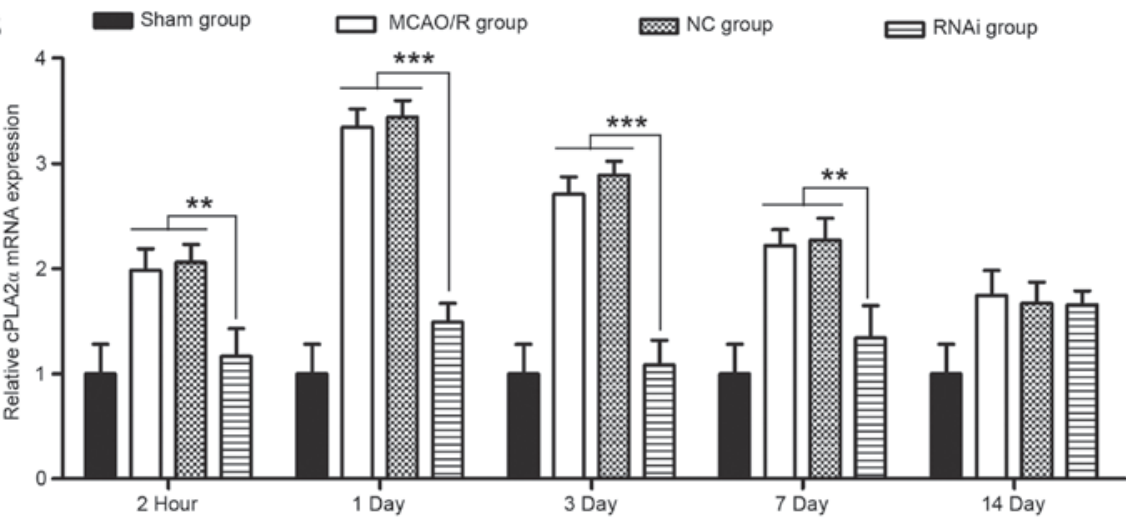

C

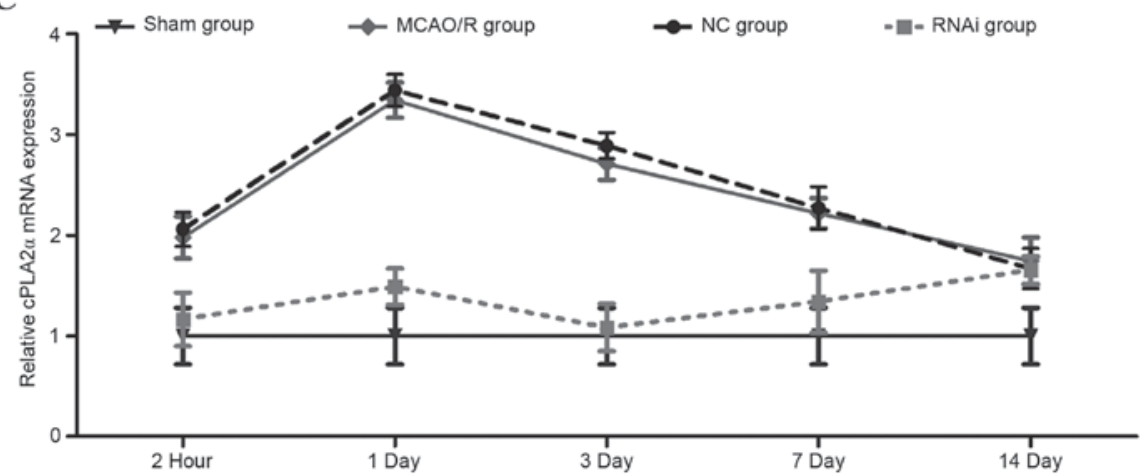

Figure 3. Expression levels of cPLA2 $\alpha$ in mice brains following MCAO/R. (A) Representative western blot analysis of cPLA2 $\alpha$ protein levels in whole brain lysates from the stroke brains of mice at $2 \mathrm{~h}$, day 1,3, 7 and 14 after the MCAO/R procedure. (B and C) Expression levels of cPLA2 $\alpha$ mRNA at different time-points following $\mathrm{MCAO} / \mathrm{R}$ surgery were detected by quantitative polymerase chain reaction. Data are presented as means \pm standard deviation of three independent experiments. ${ }^{* *} \mathrm{P}<0.01$ and ${ }^{* * *} \mathrm{P}<0.001$ (Student's t-test). cPLA2 $\alpha$, cytosolic phospholipase A2 $\alpha$; MCAO/R, middle cerebral artery occlusion and/or reperfusion; NC, negative control; RNAi, RNA interference.

the RNAi group mice exhibited a reduced level of PGE2 compared with the sham, MCAO/R and $\mathrm{NC}$ group animals at $24 \mathrm{~h}$ following surgery (Fig. 4C; $\mathrm{P}<0.001$ ). Similarly, reduced levels of LTB4 were observed in the serum of the RNAi group animals (Fig. 4D; P<0.001). PGE2 and LTB4 in the serum were significantly decreased following pAd-siRNA-cPLA2 $\alpha$ treatment (Fig. 4).

Effects of cPLA2 $\alpha$ on pathological changes. H\&E staining effectively demonstrated the pathological changes. The examination of these pathological sections demonstrated that the morphological features of the brain tissue samples were normal 14 days after surgery in the sham group (Fig. 5A), while the morphological features of the brain tissue samples from the other three groups exhibited significant changes. $\mathrm{MCAO} / \mathrm{R}$ tissue samples exhibited nerve cell necrosis, loose structures and visible intercellular edema to different degrees, which validated that the MCAO/R surgery had been successfully performed. However, compared with the $\mathrm{MCAO} / \mathrm{R}$ group or NC group, the histological structure changes of the brain tissue samples were less marked in the
RNAi group. The results demonstrate that the treatment of cPLA2 $\alpha$ RNAi ameliorated pathological changes following ischemic brain damage in mice.

Effects of cPLA2 $\alpha$ on reducing ischemia-induced brain infarct volume. Brain infarct was assessed following the experiment using laboratory standard volumetric analysis of the anterior and posterior views of coronal slabs stained with TTC, and corrected for swelling. No infarction was observed in the sham group, whereas extensive lesions were observed in the model and NC groups. RNAi treatment effectively decreased the cerebral injury with a significant reduction in infarct volume of the ischemic hemisphere when compared with the $\mathrm{NC}$ or model group mice (Fig. 5B; $\mathrm{P}<0.01$ ).

Effects of cPLA2 $\alpha$ on apoptosis in braincells. TUNEL staining was performed to evaluate whether pAd-siRNA-cPLA2 $\alpha$ treatment alleviates cell apoptosis by decreasing cPLA2 $\alpha$ expression levels (Fig. 5C and E). TUNEL ${ }^{+}$cell number in the brain tissue of mice following $\mathrm{MCAO} / \mathrm{R}$ surgery exhibited a significant increase when compared with the 

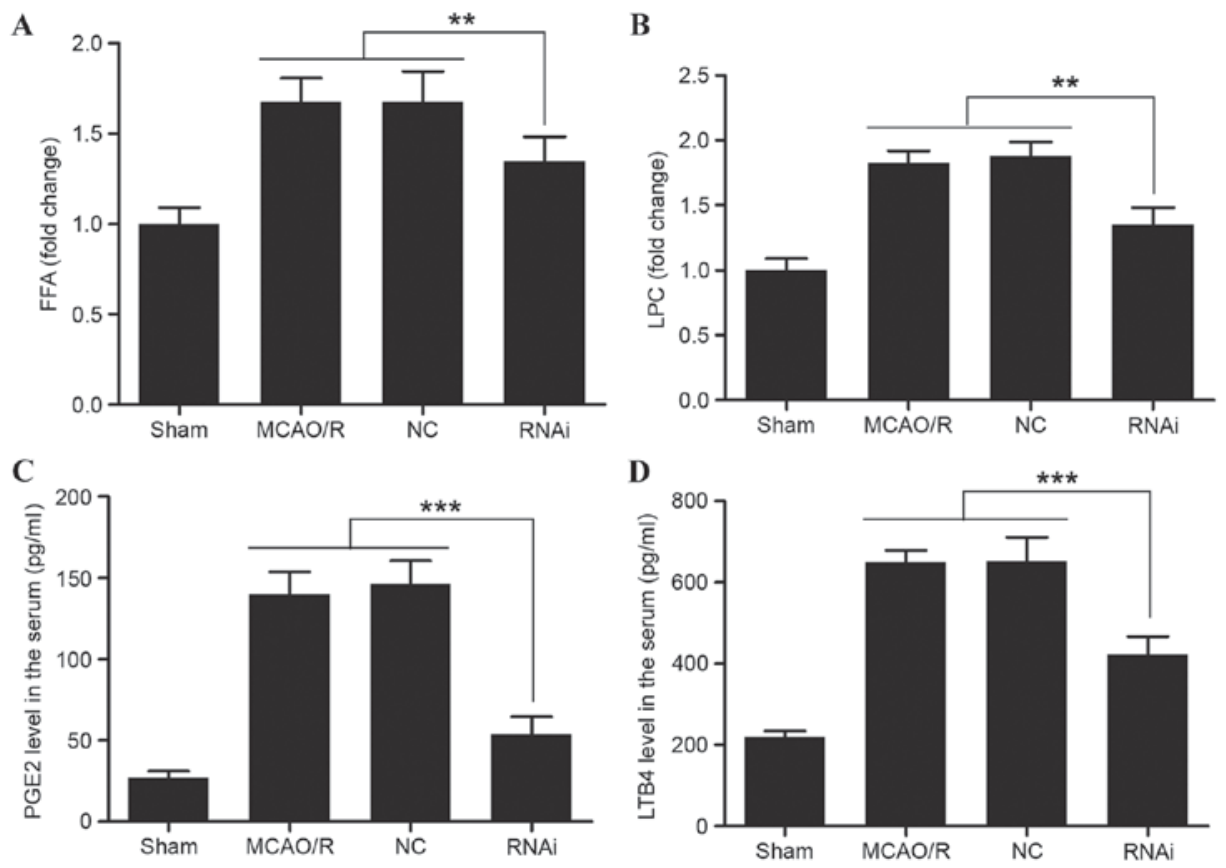

Figure 4. Effects of siRNA-cPLA2 $\alpha$ on the expression of FFA, LPC, PGE2 and LTB4 in mice after MCAO/R. Expression levels of (A) FFA and (B) LPC in the brain tissue from mice $24 \mathrm{~h}$ after MCAO/R surgery were measured by high performance thin layer chromatography. Expression levels of (C) PGE2 and (D) LTB4 in the serum of mice $24 \mathrm{~h}$ after MCAO/R surgery were measured using ELISA. pAd-siRNA-cPLA2 $\alpha$ treatment decreased the expression levels of FFA, LPC, PGE2 and LTB4. Data are presented as means \pm standard deviation of three independent experiments. ${ }^{* *} \mathrm{P}<0.01$ and ${ }^{* * *} \mathrm{P}<0.001$ (Student's t-test). siRNA, small interfering RNA; cPLA2 $\alpha$, cytosolic phospholipase A2 $\alpha$; FFA, free fatty acids; LPC, lysophosphatidylcholine; PGE2, prostaglandin E2; LTB4, leukotriene B4; MCAO/R, middle cerebral artery occlusion and/or reperfusion; NC, negative control.

sham group mice at each time-point. The RNAi group had a smaller number of TUNEL-positive cells in comparison to the NC group on days 7 and 14. Such results indicate that pAd-siRNA-cPLA2 $\alpha$ treatment may improve pathological changes by inhibiting apoptosis in mice with focal ischemic brain damage.

\section{Discussion}

The treatment of cerebral ischemia should be selected according to the principle of damage cascades. The aim of clinical treatment of cerebral ischemia is to recover the blood and oxygen supply, suppress inflammation of the ischemic area, and maintain the integrity of the neuron structure and function. In the present study, it was validated and confirmed that cPLA $2 \alpha$ significantly influences the speed of recovery from cerebral ischemia. In addition, cPLA $2 \alpha$-knockout effectively suppresses inflammation and helps to maintain the integrity of the neuron structure and function. The present results provide a reference for the potential use of adenoviruses-mediated RNAi targeting cPLA $2 \alpha$ in the clinical setting.

Numerous studies demonstrated that the level of cPLA2a was closely associated with the functional recovery in stroke (11), Alzheimer's disease (38) and multiple sclerosis (34), which indicated the causative role of cPLA2a in neurodegeneration. cPLA $2 \alpha$ has a major role in rat cerebral ischemia, and upregulated expression of cPLA $2 \alpha$ following trauma may be significant in secondary injury, such as inflammation, nociception and functional deficits (11). Certain studies indicated that certain inhibitors of cPLA $2 \alpha$ inhibit the activity of cPLA $2 \alpha$, but not the protein expression and that the effects are short term $(22,39)$. In the present study, three adenoviruses-mediated RNAi sequences were established and their effects in L929 cells were identified. An RNAi sequence that inhibits the expression and activity of mouse cPLA2 $\alpha$ was successfully identified (Fig. 1). The MCAO/R mice were treated with pAd-siRNA-cPLA2 $\alpha$ prior to injury, and it was observed that it effectively reduced the expression levels of cPLA2 $\alpha$ (Fig. 3). pAd-siRNA-cPLA2 $\alpha$ affects the expression of cPLA2 $\alpha$ in the long-term (14 days) following only one injection (Fig. 3C).

The fact that inhibition of cPLA $2 \alpha$ attenuates focal ischemic brain damage in mice indicates that the activity of cPLA $2 \alpha$ contributes markedly to the injury cascade following MCAO/R surgery $(9,10)$. The effects of cPLA $2 \alpha$ on focal ischemic brain damage go beyond the phase of acute injury. Indeed, the present and previous studies have demonstrated that the secondary injury mechanism is closely associated with cPLA $2 \alpha(11)$, as the cPLA2 $\alpha$ RNAi treatment reduced the injury following 3 days of reperfusion. It has been reported previously that upregulation of cPLA $2 \alpha$ is relevant to blood-brain barrier disruption in rats at $24 \mathrm{~h}$ of reperfusion (40).

A total of $75 \%$ of patients with cerebral ischemia exhibit varying degrees of neurological deficit (41), and motor function deficits are an important manifestation of neurological deficits (42). Neurological deficits are a characteristic of cerebral ischemia. Thus, motor function recovery is an important index for evaluating the therapeutic efficacy of medications. An apparent neurological deficit was observed from $2 \mathrm{~h}$ after $\mathrm{MCAO} / \mathrm{R}$ surgery in the present study, which validated the cerebral ischemia model. However, the finding that the NDS was reduced in the RNAi group compared with the NC group 
A

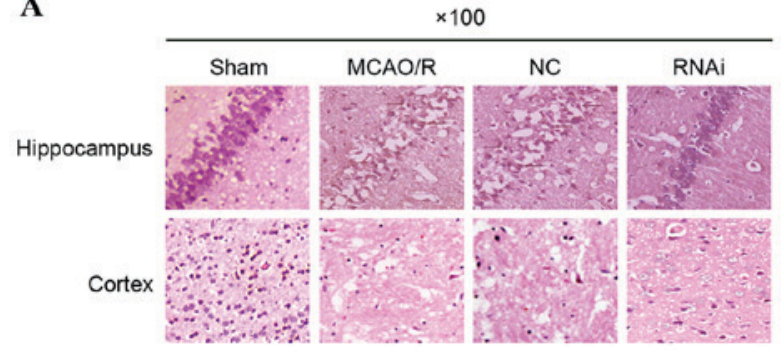

C

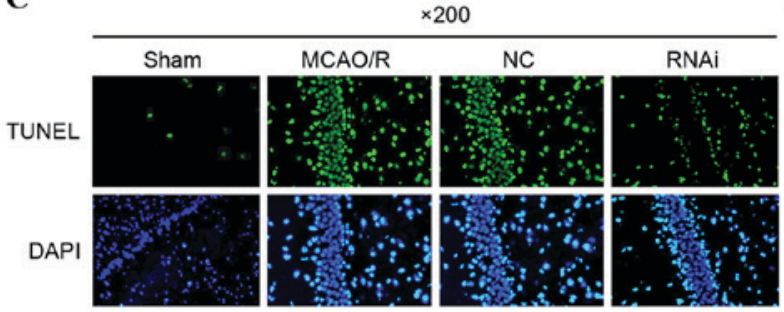

$\mathbf{E}$

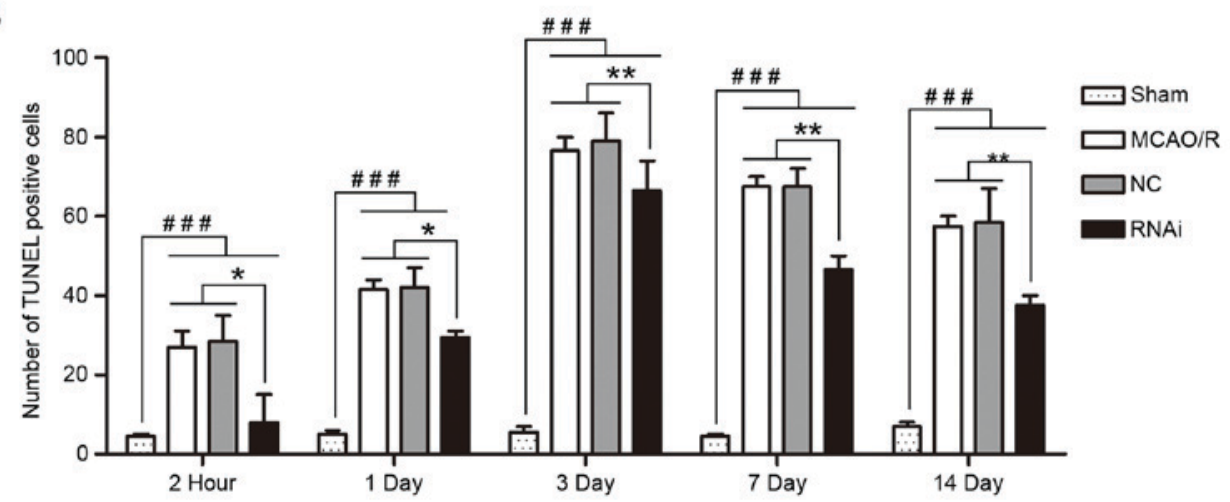

B

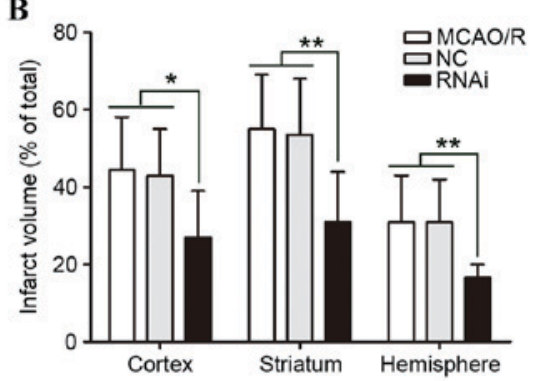

D

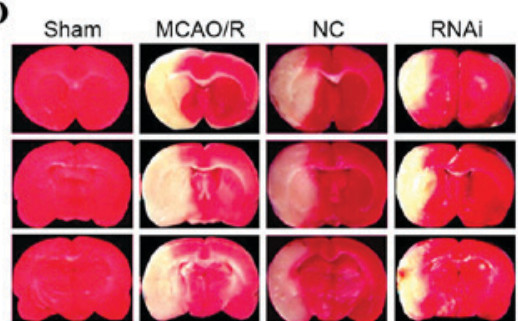

Figure 5. Effects of siRNA-cPLA2 $\alpha$ on pathological changes, cell apoptosis, and infarct volume of mice following MCAO/R. (A) Pathological changes of hippocampal and cortical tissues following pAd-siRNA-cPLA2 $\alpha$ treatment (magnification, x100). (B) PAd-siRNA-cPLA2 $\alpha$ treatment protects mice from brain infarction 14 days after MCAO/R. (C) Representative images of TUNEL staining from mice brain tissue samples 14 days after MCAO/R (magnification, x200). (D) Triphenyltetrazolium chloride staining images from mice brain tissue samples 14 days after MCAO/R. (E) Number of TUNEL-positive cells was counted. Data are presented as means \pm standard deviation of three independent experiments. ${ }^{*} \mathrm{P}<0.05$ and ${ }^{* * *} \mathrm{P}<0.01, \mathrm{RNAi}$ group vs. the MCAO/R and NC group; ${ }^{\# \# \# ~} \mathrm{P}<0.001$, sham group vs. the other three groups (Student's t-test). siRNA, small interfering RNA; cPLA2 $\alpha$, cytosolic phospholipase A2 $\alpha ; \mathrm{MCAO} / \mathrm{R}$, middle cerebral artery occlusion and/or reperfusion; RNAi, RNA interference; TUNEL, terminal deoxynucleotidyl transferase dUTP nick end-labeling; NC, negative control.

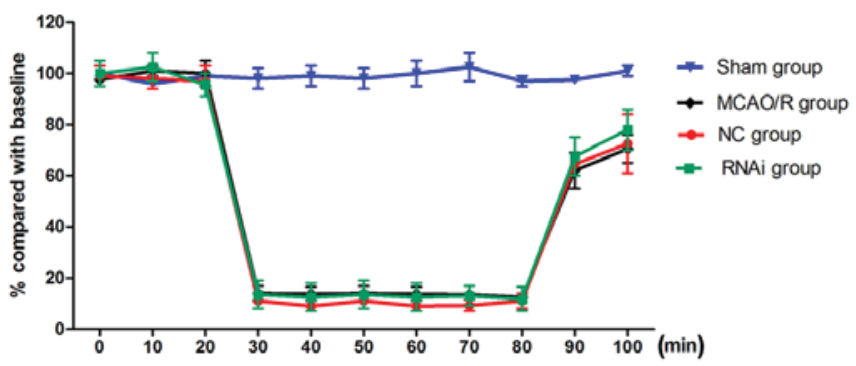

Figure 6. Effects of siRNA-cPLA2 $\alpha$ on mouse blood flow prior to and following $\mathrm{MCAO} / \mathrm{R}$. Laser-Doppler flow measurement of cerebral blood flow was performed prior to surgery $(0-20 \mathrm{~min})$, during the surgery $(20-80 \mathrm{~min})$ and in the reperfusion (80-100 $\mathrm{min}$ ) period. Data are presented as the mean \pm standard deviation of the mice in the same group. pAd-siRNA-cPLA2 $\alpha$ treatment does not alter relative cerebral blood flow. $\mathrm{MCAO} / \mathrm{R}$, middle cerebral artery occlusion and/or reperfusion; siRNA, small interfering RNA; cPLA2 $\alpha$, cytosolic phospholipase $\mathrm{A} 2 \alpha$.

supports the conclusion that pAd-siRNA-cPLA2 $\alpha$ treatment protects against functional injury (Fig. 2A). The motor function recovery was restored faster in the RNAi group, which was assessed by the number of falls by mice following MCAO/R surgery (Fig. 2B).

Inhibition of cPLA $2 \alpha$ to treat cerebral ischemia has certain anti-inflammatory and neuroprotective effects, expediting the processes involved in physiological function recovery. Previous studies regarding the link between cPLA $2 \alpha$ and cerebral ischemia have revealed that levels of cyclooxygenase (COX)-2 in the basal and stimulated central nervous system are reduced in cPLA $2 \alpha$ knock-out animals $(43,44)$. Proinflammatory lipid mediators, such as PGE2, LTB4, LPC and FFA, which are regulated by the activity of CPLA $2 \alpha$ are closely associated with ischemic brain damage. COX-2 and 5-lipoxygenase (5-LOX), as well as their products, were involved in neuron damage and neurodegeneration. FFA represents predominantly free AA, and was catalyzed to prostaglandins and leukotrienes by COX-2 and 5-LOX, respectively. In the present study, PGE2 was a representative product of COX-2, and LTB4 was a representative product of 5-LOX. LPC is an important signaling molecule correlated with chronic inflammation and 
tissue damage (45). In the present study, MCAO/R surgery mice treated with pAd-siRNA-cPLA2 $\alpha$ demonstrated effectively reduced expression levels of those proinflammatory lipid mediators (Fig. 4), indicating that they originated from cPLA $2 \alpha$. H\&E staining demonstrated that the RNAi group changes were less marked when compared with the NC group. Similarly, mice treatment with pAd-siRNA-cPLA2 $\alpha$ exhibited a decreased number of TUNEL ${ }^{+}$cells and the brain infarct volume was reduced. These findings indicate that treatment with pAd-siRNA-cPLA2 $\alpha$ may alleviate the pathological damage by reducing inflammation and inhibiting apoptosis following $\mathrm{MCAO} / \mathrm{R}$ surgery. Thus, these data suggest that treatment with pAd-siRNA-cPLA $2 \alpha$ inhibits cPLA2 $\alpha$ effectively and efficiently protects cells from ischemic-induced adverse effects.

In conclusion, the present study demonstrates the therapeutic potential of the adenoviruses-mediated RNAi targeting cPLA $2 \alpha$ in a cerebral ischemia animal model. The efficacy of the treatment was predominantly attributed to the inhibition of cPLA $2 \alpha$ and the reduction of cPLA $2 \alpha$-derived proinflammatory lipid mediators. The neuroprotective effects of pAd-siRNA-cPLA $2 \alpha$ treatment in the MCAO/R mice indicated that a long-term effect cPLA $2 \alpha$ inhibitor may be used in the future as a therapeutic strategy for cerebral ischemia. However, further investigations are required to evaluate the adenoviruses-mediated RNAi targeting cPLA2 $2 \alpha$ in human cerebral ischemia patients.

\section{Acknowledgements}

The authors would like to thank the Cangzhou Central Hospital (Hebei, China) for their support.

\section{Funding}

No funding was received.

\section{Availability of data and materials}

The analyzed datasets generated during the study are available from the corresponding author on reasonable request.

\section{Authors' contributions}

HW and LZ conceived and designed the study. HW, HL and FZ performed the experiments. HW wrote the study. LZ reviewed and edited the study. All authors read and approved the study.

\section{Ethics approval and consent to participate}

All procedures performed with mice were according to the National Institutes of Health Guide for the Care and Use of Laboratory Animals, and the experimental procedures were approved by the Ethics Committee of Cangzhou Central Hospital (Hebei, China).

\section{Consent for publication}

Not applicable.

\section{Competing interests}

The authors declare that they have no competing interests.

\section{References}

1. Arai K, Ikegaya Y, Nakatani Y, Kudo I, Nishiyama N and Matsuki N: Phospholipase A2 mediates ischemic injury in the hippocampus: A regional difference of neuronal vulnerability. In Eur J Neurosci 13: 2319-2323, 2001.

2. Bonventre JV, Huang Z, Taheri MR, O'Leary E, Li E, Moskowitz MA and Sapirstein A: Reduced fertility and postischaemic brain injury in mice deficient in cytosolic phospholipase A2. Nature 390: 622-625, 1997.

3. Brady KM, Texel SJ, Kishimoto K, Koehler RC and Sapirstein A: Cytosolic phospholipase A alpha modulates NMDA neurotoxicity in mouse hippocampal cultures. Eur J Neurosci 24: 3381-3386, 2006

4. Ward NC, Croft KD, Blacker D, Hankey GJ, Barden A, Mori TA, Puddey IB and Beer CD: Cytochrome P450 metabolites of arachidonic acid are elevated in stroke patients compared with healthy controls. Clin Sci (Lond) 121: 501-507, 2011.

5. Moskowitz MA, Lo EH and Iadecola C: The science of stroke: Mechanisms in search of treatments. Neuron 67: 181-198, 2010.

6. Menschikowski M, Hagelgans A and Siegert G: Secretory phospholipase A2 of group IIA: Is it an offensive or a defensive player during atherosclerosis and other inflammatory diseases? Prostaglandins Other Lipid Mediat 79: 1-33, 2006.

7. Gabryel B, Chalimoniuk M, Stolecka A and Langfort J: Activation of cPLA2 and sPLA2 in astrocytes exposed to simulated ischemia in vitro. Cell Biol Int 31: 958-965, 2007.

8. Williams SD and Gottlieb RA: Inhibition of mitochondrial calcium-independent phospholipase A2 (iPLA2) attenuates mitochondrial phospholipid loss and is cardioprotective. Biochem J 362: 23-32, 2002.

9. Kishimoto K, Li RC, Zhang J, Klaus JA, Kibler KK, Doré S, Koehler RC and Sapirstein A: Cytosolic phospholipase A2 alpha amplifies early cyclooxygenase-2 expression, oxidative stress and MAP kinase phosphorylation after cerebral ischemia in mice. J Neuroinflammation 7: 42, 2010.

10. Shen Y, Kishimoto K, Linden DJ and Sapirstein A: Cytosolic phospholipase A(2) alpha mediates electrophysiologic responses of hippocampal pyramidal neurons to neurotoxic NMDA treatment. Proc Natl Acad Sci USA 104: 6078-6083, 2007.

11. Zhang J, Barasch N, Li RC and Sapirstein A: Inhibition of cytosolic phospholipase $\mathrm{A}(2)$ alpha protects against focal ischemic brain damage in mice. Brain Res 1471: 129-137, 2012.

12. Leung RK and Whittaker PA: RNA interference: From gene silencing to gene-specific therapeutics. Pharmacol Ther 107: 222-239, 2005.

13. Elbashir SM, Harborth J, Lendeckel W, Yalcin A, Weber K and Tuschl T: Duplexes of 21-nucleotide RNAs mediate RNA interference in cultured mammalian cells. Nature 411: 494-498, 2001.

14. Dykxhoorn DM and Lieberman J: The silent revolution: RNA interference as basic biology, research tool, and therapeutic. Annu Rev Med 56: 401-423, 2005.

15. Morris KV and Rossi JJ: Lentiviral-mediated delivery of siRNAs for antiviral therapy. Gene Ther 13: 553-558, 2006.

16. Oualikene W, Lamoureux L, Weber JM and Massie B: Protease-deleted adenovirus vectors and complementing cell lines: Potential applications of single-round replication mutants for vaccination and gene therapy. Hum Gene Ther 11: 1341-1353, 2000.

17. Abea K, Setoguchib Y, Hayashia T and Itoyama Y: In vivo adenovirus-mediated gene transfer and the expression in ischemic and reperfused rat brain. Brain Res 763: 191-201, 1997.

18. Shimizu M, Matsumoto Y, Kurosawa T, Azuma C, Enomoto M, Nakamura H, Hirabayashi T, Kaneko M, Okuma Y and Murayama T: Release of arachidonic acid induced by tumor necrosis factor-alpha in the presence of caspase inhibition: Evidence for a cytosolic phospholipase A2alpha-independent pathway. Biochem Pharmacol 75: 1358-1369, 2008.

19. Shen C, Buck AK, Liu X, Winkler M and Reske SN: Gene silencing by adenovirus-delivered siRNA. FEBS Lett 539: 111-114, 2003.

20. Sambrook J, Fritsch EF and Maniatis T: Molecular Cloning: A Laboratory Manual (2nd ed). Cold Spring Harbor Laboratory Press, New York, 1989. 
21. National Research Council: Guide for the Care and Use of Laboratory Animals. The National Academies Press, Washington DC, 1996. https://doi.org/10.17226/5140 Accessed on September 6, 2014

22. Zhang J, Barasch N, Li RC and Sapirstein A: Inhibition of cytosolic phospholipase $\mathrm{A}(2)$ alpha protects against focal ischemic brain damage in mice. Brain Res 1471: 129-137, 2012.

23. Longa EZ, Weinstein PR, Carlson S and Cummins R: Reversible middle cerebral artery occlusion without craniectomy in rats. Stroke 20: 84-91, 1989.

24. Khan M, Singh J and Singh I: Plasmalogen deficiency in cerebral adrenoleukodystrophy and its modulation by lovastatin. J Neurochem 106: 1766-1779, 2008

25. Khan M, Contreras M and Singh I: Endotoxin-induced alterations of lipid and fatty acid compositions in rat liver peroxisomes. J Endotoxin Res 6: 41-50, 2000.

26. Weerheim AM, Kolb AM,Sturk A and Nieuwland R: Phospholipid composition of cell-derived microparticles determined by one-dimensional high-performance thin-layer chromatography. Anal Biochem 302: 191-198, 2002.

27. Weissman L, Jo DG, Sorensen MM, de Souza-Pinto NC, Markesbery WR, Mattson MP and Bohr VA: Defective DNA base excision repair in brain from individuals with Alzheimer's disease and amnestic mild cognitive impairment. Nucleic Acids Res 35: 5545-5555, 2007.

28. Bradford MM: A rapid and sensitive method for the quantitation of microgram quantities of protein utilizing the principle of protein-dye binding. Anal Biochem 72: 248-254, 1976.

29. Hu N, Zhang J, Cui W, Kong G, Zhang S, Yue L, Bai X, Zhang Z, Zhang W, Zhang $X$ and Ye L: miR-520b regulates migration of breast cancer cells by targeting hepatitis B X-interacting protein and interleukin-8. J Biol Chem 286: 13714-13722, 2011.

30. Livak KJ and Schmittgen TD: Analysis of relative gene expression data using real-time quantitative PCR and the 2(Delta Delta C(T)) method. Methods 25: 402-408, 2001.

31. Arumugam TV, Phillips TM, Cheng A, Morrell CH, Mattson MP and Wan R: Age and energy intake interact to modify cell stress pathways and stroke outcome. Ann Neurol 67: 41-52, 2010.

32. Swanson RA and Sharp FR: Infarct measurement methodology. J Cereb Blood Flow Metab 14: 697-698, 1994.

33. Ackermann EJ, Conde-Frieboes K and Dennis EA: Inhibition of macrophage $\mathrm{Ca}(2+)$-independent phospholipase $\mathrm{A} 2$ by bromoenol lactone and trifluoromethyl ketones. J Biol Chem 270: 445-450, 1995

34. Kalyvas A and David S: Cytosolic phospholipase A2 plays a key role in the pathogenesis of multiple sclerosis-like disease. Neuron 41: 323-335, 2004.

35. Myou S, Sano H, Fujimura M, Zhu X, Kurashima K, Kita T, Nakao S, Nonomura A, Shioya T, Kim KP, et al: Blockade of eosinophil migration and airway hyperresponsiveness by cPLA2-inhibition. Nat Immunol 2: 145-149, 2001.
36. Nagase T, Uozumi N, Aoki-Nagase T, Terawaki K, Ishii S, Tomita T, Yamamoto H, Hashizume K, Ouchi Y and Shimizu T: A potent inhibitor of cytosolic phospholipase A2, arachidonyl trifluoromethyl ketone, attenuates LPS-induced lung injury in mice. Am J Physiol Lung Cell Mol Physiol 284: L720-L726, 2003.

37. Riendeau D, Guay J, Weech PK, Laliberte F, Yergey J, Li C, Desmarais S, Perrier H, Liu S, Nicoll-Griffith D, et al: Arachidonyl trifluoromethyl ketone, a potent inhibitor of $85-\mathrm{kDa}$ phospholipase A2, blocks production of arachidonate and 12-hydroxyeicosatetraenoic acid by calcium ionophore-challenged platelets. J Biol Chem 269: 15619-15624, 1994.

38. Sanchez-Mejia RO, Newman JW, Toh S, Yu GQ, Zhou Y, Halabisky B, Cissé M, Scearce-Levie K, Cheng IH, Gan L, et al: Phospholipase A2 reduction ameliorates cognitive deficits in a mouse model of Alzheimer's disease. Nat Neurosci 11: 1311-1318, 2008.

39. Khan M, Shunmugavel A, Dhammu TS, Matsuda F, Singh AK and Singh I: Oral administration of cytosolic PLA2 inhibitor arachidonyl trifluoromethyl ketone ameliorates cauda equina compression injury in rats. J Neuroinflammation 12: 94, 2015.

40. Nito C, Kamada H, Endo H, Niizuma K, Mye DJ and Chan PH: Role of the p38 mitogen-activated protein kinase/cytosolic phospholipase A2 signaling pathway in bolld-brain barrier disruption after focal cerebral ischemia and reperfusion. J Cereb Blood Flow Metab 28: 1686-1696, 2008.

41. Cramer SC: Repairing the human brain after stroke. II. Restorative therapies. Ann Neurol 63: 549-560, 2008.

42. Cramer SC and Crafton KR: Somatotopy and movement representation sites following cortical stroke. Exp Brain Res 168: 25-32, 2006.

43. Bosetti F and Weerasinghe GR: The expression of brain cyclooxygenase- 2 is down-regulated in the cytosolic phospholipase A2 knockout mouse. J Neurochem 87: 1471-1477, 2003

44. Sapirstein A, Saito H, Texel SJ, Samad TA, O'Leary E and Bonventre JV: Cytosolic phospholipase A2alpha regulates induction of brain cyclooxygenase- 2 in a mouse model of inflammation. Am J Physiol Regul Integr Comp Physiol 288: R1774-R1782, 2005.

45. Sevastou I, Kaffe E, Mouratis MA and Aidinis V: Lysoglycerophospholipids in chronic inflammatory disorders: The PLA(2)/LPC and ATX/LPA axes. Biochim Biophys Acta 1831: 42-60, 2013.

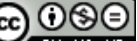

This work is licensed under a Creative Commons Attribution-NonCommercial-NoDerivatives 4.0 International (CC BY-NC-ND 4.0) License. 